\title{
NOUVELLE
}

\section{Greatwall, un nouveau gardien de la mitose}

Aicha Gharbi-Ayachi, Andrew Burgess, Suzanne Vigneron, Jean-Claude Labbé, Anna Castro, Thierry Lorca
Centre de recherche de biochimie macromoléculaire (CRBM), 1919 , route de Mende,

34293 Montpellier, France.

anna.castro@crbm.cnrs.fr,

thierry.lorca@crbm.cnrs.fr

\section{Schéma classique : l'entrée en mitose} dépend de l'activation de Cdkl-cycline B L'entrée en mitose repose sur l'activation de la kinase mitotique Cdkl (cyclindependent kinase 1)-cycline B. Au cours de la phase $\mathrm{G} 2$, la sous-unité cycline $B$ est synthétisée et s'associe à sa sous-unité catalytique Cdkl. Le complexe Cdklcycline $B$ ainsi formé est maintenu inactif par la phosphorylation de la sous-unité catalytique $C d k 1$ sur les résidus Thrl4 et Tyr15 par les kinases Mytl et Weel [11]. Ces deux kinases sont actives au cours de la phase G2 sous une forme déphosphorylée et s'inactivent en mitose par phosphorylation. À la transition G2-M, les phosphatases de la famille Cdc25 déphosphorylent les deux résidus Thr14 et Tyrl5 inhibiteurs permettant l'activation de la kinase Cdkl-cycline B et l'entrée en phase $M$ du cycle cellulaire. Une fois complètement activée, $C d k 1$-cycline $B$ phosphoryle de nombreux substrats requis pour le bon déroulement de la mitose. Le modèle unanimement accepté propose qu'en fin de phase G2, la déphosphorylation de la Thrl4 et de la Tyrl5 de Cdkl par une fraction active de $\mathrm{Cdc} 25$ induit une activation initiale du complexe Cdkl-cycline B, qui à son tour active pleinement $\mathrm{Cdc} 25$ et inactive Mytl et Weel par phosphorylation. Ces événements rapides induisent une activation maximale du complexe Cdkl-cycline $B$ et une entrée irréversible en mitose. Ce mécanisme de régulation est communément appelé boucle d'amplification du MPF (M phase promoting factor). Toutefois, ce modèle a récemment évolué grâce à de nouvelles données montrant qu'en plus du complexe $(d k 1$-cycline B, la protéine Greatwall ( $\mathrm{Gwl}$ ) est aussi requise pour entrer en mitose et maintenir l'état mitotique [1].

Greatwall, un second chef d'orchestre qui inhibe la déphosphorylation des substrats mitotiques

Gwl est une protéine initialement caractérisée lors d'une recherche de mutants de drosophile présentant des défauts de condensation des chromosomes [2]. Gwl est une protéine kinase dont la fonction est encore peu documentée. Elle présente l'originalité d'une structure de son domaine kinase séparée en deux parties par une séquence de plus de 500 acides aminés. Le gène $G w l$ est conservé de la drosophile jusqu'à l'homme. Les premières études réalisées chez le xénope montrent que l'élimination de la protéine kinase Gwl induit une inhibition de l'entrée en phase M ou bien une sortie prématurée de mitose [3]. Ces travaux suggèrent que Gwl est requise pour maintenir l'état mitotique en jouant un rôle dans la boucle d'amplification du MPF. L'hypothèse la plus probable est que Gwl participe à l'activation de la protéine phosphatase $\mathrm{Cdc} 25$ ou bien à une régulation négative des protéines kinases Weel et/ou Mytl. Toutefois, nos récents résultats montrent que le rôle principal de Gwl ne réside pas dans cette voie d'amplification du MPF mais plutôt dans l'inhibition de PP2A, une phosphatase qui déphosphoryle les substrats du complexe Cdkl-cycline B [4]. L'importance de nos données, confirmées par d'autres laboratoires [5], repose sur une observation surprenante qui montre pour la première fois qu'en présence d'une forte activité kinase du complexe Cdkl-cycline B, l'élimination de Gwl empêche la phosphoryla- tion des substrats de la protéine kinase Cdkl-cycline B, entraînant une sortie de mitose prématurée. Jusqu'à présent, l'entrée en mitose était directement liée à l'activation de la kinase mitotique Cdklcycline B. Aujourd'hui, nous apportons un nouvel élément à l'édifice mitotique avec $\mathrm{Gwl}$ comme autre chef d'orchestre qui contrôle négativement l'activité de PP2A. Ce nouveau concept établit que l'entrée en mitose repose dorénavant sur l'activation conjointe de deux kinases, Cdklcycline $B$ et $\mathrm{Gwl}$, la première permettant la phosphorylation massive des substrats mitotiques requis pour l'entrée en mitose, la seconde inhibant la phosphatase PP2A qui déphosphoryle ces mêmes substrats (Figure 1) [4, 5]. Plus récemment, nous avons identifié par complémentation chez le xénope l'homologue humain de la protéine kinase $\mathrm{Gwl}$ [6]. Il s'agit de la protéine MAST-L (pour microtubule associated serine/threonine kinase-like). L'analyse du rôle de Gwl dans les cellules humaines met en évidence une voie de signalisation conservée avec des défauts mitotiques majeurs en l'absence de Gwl. La voie mitotique est restaurée soit par complémentation avec la protéine kinase Gwl de xénope, soit par l'inhibition de la PP2A. Nos résultats montrent qu'une élimination totale de Gwl dans les cellules HeLa entraîne un arrêt des cellules en phase G2. Toutefois, si Gwl n'est pas parfaitement éliminée, les cellules entrent en mitose et développent des défauts plus ou moins sévères. On peut noter des défauts d'attachement des chromosomes sur le fuseau mitotique, des problèmes de condensation de l'ADN et des cytokinèses aberrantes [6]. 

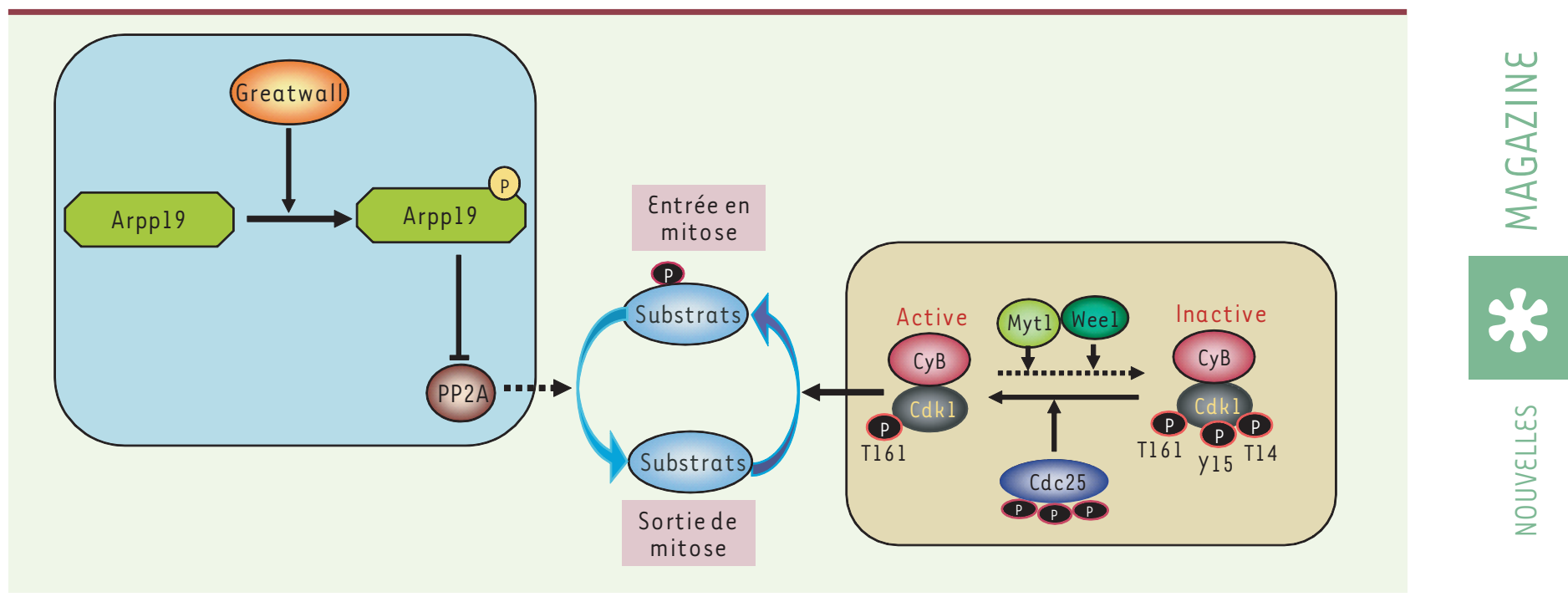

Figure 1. Arpp19, un nouveau substrat de la kinase Greatwall. L'entrée en mitose repose maintenant sur un nouveau concept qui nécessite l'activation conjointe de deux kinases, Cdkl-cycline B et Greatwall : la première permet la phosphorylation massive des substrats mitotiques (carré jaune à droite), la deuxième phosphoryle Arpp19 qui lie et inhibe la phosphatase PP2A responsable de la déphosphorylation de ces mêmes substrats (carré bleu à gauche), l'ensemble autorisant le maintien de ces phosphorylations requises pour la réalisation d'une mitose correcte. La sous-unité catalytique Cdkl est phosphorylée au niveau des résidus Thr 14 et Tyr 15 par les kinases Mytl et Weel. Les phosphatases de la famille Cdc25 déphosphorylent les deux résidus Thr14 et Tyr 15 inhibiteurs.

\section{Caractérisation du mécanisme d'action de Greatwall}

Toutefois, il restait à comprendre comment Gwl contrôle l'activité de PP2A. C'est ce que nous avons réussi à réaliser en caractérisant deux substrats de Gwl appartenant à une même famille de protéines: Arppl9 (cAMP-regulated phosphoprotein 19) et ENSA (alpha endosulfine). Le rôle de ces deux protéines n'est pas clairement identifié. Arppl9 est spécifiquement enrichie dans le cerveau mais son rôle n'y est pas connu. En ce qui concerne ENSA, une fonction a tout d'abord été mentionnée dans les cellules $\beta$ du pancréas où elle contrôle la libération d'insuline en régulant l'activité des canaux potassiques dépendant de l'ATP. D'autre part, des ovocytes de drosophile dans lesquels la séquence du gène codant pour ENSA est altérée présentent des défauts mitotiques avec une prophase prolongée et une incapacité à progresser en métaphase. De manière très remarquable, ces ovocytes possèdent une activité $C d k l$-cycline $B$ normale mais un niveau de phosphorylation des substrats mitotiques étonnamment faible [7]. Ce phénotype n'est pas sans rappeler celui que nous avons obtenu en éliminant la protéine kinase Gwl dans les extraits d'ovocyte de xénope $[4,8]$.

Nos résultats montrent que ENSA et Arppl9 sont phosphorylées in vitro par Gwl sur un site présent chez tous les membres de cette famille de la levure jusqu'à l'homme $[9,10]$. L'expression ectopique de ces deux protéines phosphorylées par Gwl est capable d'induire l'entrée en mitose et cela en présence ou en l'absence de Gwl. ENSA et Arpp19 s'associent à PP2A et inhibent son activité phosphatase qui déphosphoryle les substrats de Cdkl-cycline B (Figure 1). La phosphorylation de ces deux protéines est corrélée à l'activation de Gwl. Enfin, seule la forme endogène de la protéine Arppl9 semble jouer un rôle lors de la mitose puisque l'élimination d'Arpp19 et non pas celle d'ENSA bloque l'entrée en phase $M$ et qu'en condition de mitose l'absence d'Arppl9 ne permet plus le maintien de l'état mitotique. Cette différence fonctionnelle entre ces deux protéines pourrait être attribuée à la quasi-absence de la forme ENSA phosphorylée au cours de la mitose [9].

\section{Conclusion}

L'observation majeure faite au cours de ces travaux récents est celle de l'existence d'une nouvelle voie de régulation mettant en jeu la protéine kinase Gwl et son substrat Arppl9 comme inhibiteurs de la protéine phosphatase PP2A. Toutefois, de nombreuses questions restent sans réponse: quand et comment $\mathrm{Gwl}$ s'active-t-elle ? quels sont les complexes de PP2A inhibés par Arpp19? quel est le rôle physiologique de ENSA ? Autant de questions dont les réponses permettront d'appréhender plus précisément l'ensemble des mécanismes mis en jeu lors de la division cellulaire. $\diamond$ Greatwall, a new guardian of mitosis

\section{CONFLIT D'INTÉRÊTS}

L'auteur déclare n'avoir aucun conflit d'intérêts concernant les données publiées dans cet article.

\section{REMERCIEMENTS}

Ces travaux ont pu être réalisés grâce au soutien financier de l'ARC (Association pour la 
recherche sur le cancer) et de la Ligue nationale contre le cancer (Languedoc Roussillon, Comité $d u$ Gard). Andrew Burgess reçoit un financement de la Fondation pour la recherche médicale.

\section{RÉFÉRENCES}

1. Jackson PK. Climbing the Greatwall to mitosis. Mol Cell 2006 ; 22 : 156-7.

2. $\mathrm{Yu}_{\mathrm{u}}$, Fleming SL, Williams B, et al. Greatwall kinase : a nuclear protein required for proper chromosome condensation and mitotic progression in Drosophila. J Cell Biol $2004 ; 164$ : 487-92.

3. Yu J, Zhao Y, Li Z, et al. Greatwall kinase participates in the Cdc2 autoregulatory loop in Xenopus egg extracts. Mol Cell $2006 ; 22: 83-91$.
4. Vigneron $S$, Brioudes $\varepsilon$, Burgess A, et al. Greatwall maintains mitosis through regulation of PP2A. EMBO $2009 ; 28: 2786-93$.

5. Castilho PV, Williams BC, Mochida S, et al. The M phase kinase Greatwall $(\mathrm{Gwl})$ promotes inactivation of PP2A/B55delta, a phosphatase directed against CDK phosphosites. Mol Biol Cell $2009 ; 20: 4777-89$.

6. Burgess A, Vigneron $S$, Brioudes $\varepsilon$, et al. Loss of human Greatwall results in $\mathrm{G} 2$ arrest and multiple mitotic defects due to deregulation of the cyclin B Cdc2/PP2A balance. Proc Natl Acad Sci USA 2010 ; 107 : 12564-9.

7. Von Stetina JR, Tranguch S, Dey SK, et al. AlphaEndosulfine is a conserved protein required for oocyte meiotic maturation in Drosophila. Development 2008 ; $35: 3697-706$.

8. Lorca T, Bernis C, Vigneron S, et al. Constant regulation of the MPF amplification loop and of the Greatwall/PP2A pathway is required for metaphase

\section{NOUVELLE}

\section{Attaque bactérienne du système ubiquitine- protéasome}

Julien Burger, Jorge Merlet, Lionel Pintard
II-arrest and for a correct entry into first embryonic cell cycle.J Cell Sci 2010 ; 123 : 2281-91.

9. Gharbi-Ayachi A, Labbé JC, Burgess A, et al. The Greatwall substrate Arpp 19 controls mitosis by inhibiting PP2A. Science $2010 ; 17$ : 1673-7.

10. Mochida S, Maslen SL, Skehel M, et al. Greatwall phosphorylates an inhibitor of protein phosphatase $2 \mathrm{~A}$ that is essential for mitosis. Science $2010 ; 17$ : 1670-3.

11. Dorée M. Le déclenchement de la mitose chez les eucaryotes supérieurs. Med Sci (Paris) 2003 ; 19 : 299307.
Institut Jacques Monod, CNRS, Université Paris Diderot, Bâtiment Buffon, 15 , rue Hélène Brion, 75205 Paris Cedex 13, France. pintard.lionel@ijm.univ-paris-diderot.fr

[3] (Figure 1). Ces complexes sont les promoteurs du système ubiquitine-protéasome puisqu'ils contrôlent la dégradation d'une myriade de protéines régulatrices et notamment de nombreux régulateurs du cycle cellulaire comme p2 $7^{\text {kipl}}$, un inhibiteur de Cdk (cyclin dependent kinase), la protéine Cdtl (chromatin licensing and DNA replication factor 1) qui est dégradée en phase $S$ ce qui assure une seule réplication de I'ADN par cycle cellulaire, ou encore la GTPase RhoA qui régule le cytosquelette d'actine (Figure 1). Bien que la composition des complexes soit variable, ils sont régulés par des mécanismes identiques qui impliquent la conjugaison de la protéine Nedd8 (neural precursor cell expressed, developmentally down-regulated 8), une protéine similaire à l'ubiquitine, sur une lysine carboxy-terminale de la sous-unité culline (Figure 2). Cette modification, également appelée neddylation, induit un changement de conformation du site catalytique per- 